\title{
SPECTROPHOTOMETRIC ANALYSIS OF THE EFFECT OF AGING ON COLOR CHANGE OF MAXILLOFACIAL SILICONE
}

\author{
Aya Mohamed Fawzy* and Nada Sherin El Khourazaty**
}

\begin{abstract}
Purpose: The purpose of this study is to estimate the change in color stability of maxillofacial silicon after exposing it to natural weathering and disinfection using neutral soap (shampoo).

Material and Methods: 60 specimens were prepared from addition linking vinyl polydimethyl siloxane and divided into 3 groups $(n=20)$. The specimens were then conditioned differently as follows, Group I: Dark room storage (control group); Group II: Neutral soap disinfection; Group III: Outdoor natural weathering for 2 months. The color changes of the specimens were measured using a Reflective spectrophotometer (X-Rite, model RM200QC, Neu-Isenburg, Germany) using the Commission Internationale de I'Eclairage (CIE). ${ }^{5}$ The measurements were taken after 1 month then after two months (follow up period).
\end{abstract}

Results: There was insignificance increase in color change in neutral soap (shampoo) group. There was significance increase in color change in natural weathering group. In addition, there was significance difference between groups after 1 month and two months.

Conclusions: Exposure to outdoor natural weathering and the disinfection with neutral soap (shampoo) causes color alteration of the maxillofacial silicone.

KEYWORDS: Color change, maxillo-facial silicone, spectrophotometer, outdoor weathering, neutral soap.

\section{INTRODUCTION}

Maxillofacial prosthetics has developed through the years with great revolution in the field of prosthodontics rendering solutions to restore facial disfigurements associated with congenital deformities, acquired surgical defects, and trauma. The ultimate esthetic result of the maxilofacial prosthesis depends on the considerate regarding values of facial harmony, biocompatibility, adaptation, durability, color mixture, retention and the weight of the prosthesis. Among the several contributing reasons, the properties of the prosthetic material play a fundamental role in the ultimate result of the prosthetic treatment. The main challenge in the performance of an ideal facial prosthesis is the

\footnotetext{
* Lecturer of Prosthodontics, Faculty of Oral and Dental Medicine, Future University.

** Associate Professor of Prosthodontics, Faculty of Oral and Dental Medicine, Cairo University.
} 
degradation in appearance, either due to changes in color or deterioration of the physical properties. ${ }^{1-3}$

A large number of people acquire facial defects, unfortunately, because of ablative surgery, trauma, and congenital deformity. Facial prosthesis should restore the normal function and anatomy, protect the tissues of the defect, and provide great benefits to the patients allowing improvement in selfesteem aiding the patient to lead as normal life as possible. The most significant aim is fabrication of a prosthesis with optimal esthetics and function. Reestablishment of facial defects is a challenging task mutually between plastic surgeon and the prosthodontist. The insufficient materials existing for restoring facial defects, movable tissue beds, and difficulty in prosthesis retention furthermore the patient's approval restrict the prosthodontist. Silicones have 50 years of safety and competence in their application. Despite their development over the past 4 decades, most frequently used silicones still have some disadvantages, such as color change with time, technique sensitivity, deficiency of reparability, extrinsic color peel/fade, and lack of permanence. ${ }^{4,5}$

Degradation in appearance due to discoloration and physical properties is the main cause for replacement of facial prostheses. Frequent replacement of the prosthesis becomes less costeffective. Hygienic maintenance of maxillofacial prosthesis is of supreme significance in preserving the healthy condition of the tissues underlying the prosthesis. Mixed microbial flora including bacteria and yeast was found to colonize facial prosthesis. Disinfection of the prosthesis from 3 to 5 minutes every day is mandatory. Numerous techniques for cleansing and disinfection of the prosthesis are applied. For instance, using a lax scrubbing brush, disinfection by chemicals, or even exposure to microwave cycles. However, scrubbing alone by a brush is an insufficient technique to eradicate the bacteriological weight; subsequently sterilization by chemicals is the optimal technique for scrubbing the maxillofacial prosthesis. The properties of the silicone and the underlying tissues should not be adversely affected by the disinfecting chemical solution. ${ }^{4,6-8}$

The increasing incidence of oral cancer is the reason for the advanced research in various maxillofacial materials. Silicone elastomers chemically termed as polydimethyl siloxane have been the material of choice. It has been widely reported as better serviceable material for maxillofacial applications because of their chemical inertness, durability, strength, and ease of handling and manipulation. ${ }^{1}$

The most significant parameter in the evaluation of facial prostheses is color. Color is the major concern for the patient whether using the prosthesis or dislike and switch to a new prosthesis. However, color stability persists for only a short period of time. Alteration of the physical properties is the main reason for color fading and disliking their prostheses. Exposure to ultraviolet (UV) light, air pollution, temperature and humidity changes are the deteriorating factors. Many studies have reported that, irrespective of the kind of elastomer used in the manufacture of a maxillofacial prosthesis, its service life is usually 6 months to 2 years, with an average time of 10-12 months. ${ }^{9}$

In order to camouflage the prosthesis with shades of tissues surrounding the defect site, several pigments, rayon fiber flocking, and opacifiers are available to enhance a more realistic effect. Colorants are available in two types: inorganic and organic. The molecules of the inorganic colorants are very stable because of their ionic bonds. Before polymerization, the pigments are combined into the material and ultimate customization is enriched by painting a thin layer of pigmented adhesive onto the surface of a prostheses. Coloring the prosthesis can be intrinsic, extrinsic, or a combination of these two coloration approaches. Applying various colors in multiple layers on to the textured surface of 
the mold, building up the structure is the intrinsic coloration process. For extrinsic coloration, coloring the prosthesis is done by applying to the other surface's heterogeneities. ${ }^{10}$

The Commission Internationale de l'Eclairage (International Commission on Illumination) (CIE) $\mathrm{L}^{*} \mathrm{a} \mathrm{b}^{*}$ color difference formula is considered to present a numeric data $\left(\Delta \mathrm{E}^{*}\right)$ that provide the magnitude of the color difference perceived between 2 objects. To measure color and changes in the color of objects, the CIE presented a technique that serves as a standard language. Regarding to the Munsell color space, color is frequently designated in terms of hue, value, and chroma. Hue is the quality of a color that permits the dentist to discriminate between various families of color, value indicates the lightness of a color, and chroma is the degree of color saturation. The CIE L*a*b* color scale consists of a color space organized in a method comparable to the Munsell color space. The 3 dimensions, $\mathrm{L}^{*} \mathrm{a}^{*} \mathrm{~b}^{*}$, can explain any color in space. The CIE $\mathrm{L}^{*} \mathrm{a}^{*} \mathrm{~b}^{*}$ color difference formula is considered to represent a numeric data $\left(\Delta \mathrm{E}^{*}\right)$ that provides the magnitude of color difference perceived between 2 objects $^{11,12}$

\section{MATERIAL AND METHODS}

In this study addition linking vinyl polydimethyl siloxane room temperature silicone* was used to produce soft prosthesis. The silicone is applied either using the mixing cannul a or directly from the cartridge onto a suitable pad (glass plate, coated paper, etc.) into the molds to obtain the specimens. The material was left to completely harden without heating for 12 hours. Sixty (60) specimen were prepared then classified into three groups $(n=20)$. The specimens were conditioned differently as follows: Group I: the specimens were stored in a dark at room temperature $\left(23 \pm 2^{\circ} \mathrm{C}\right)$, Group II: Disinfection with Neutral soap (shampoo) ${ }^{* *}$, was carried out three times daily for 3 to 5 minutes each and Group III: The specimens were subjected to outdoor natural weathering. This continued for all the specimens for 2 months. The measurements were taken after 1 month then after two months (follow up period).

The conditioning periods were designated to simulate silicone prosthesis in service for 18-24 months. As each day patients wear their prosthesis approximately for $8-12 \mathrm{~h}$, through that time it is likely to be exposed at least $6 \mathrm{~h}$ of daylight, continuous sebum, perspiration and normal environmental conditions, whereas the prosthesis is on the defect site. Furthermore, before sleeping, patients spend an average of 5 min cleaning their prostheses. Therefore, 1 month of service equals $180 \mathrm{~h}$ of daylight aging, storage in sebum or acidic solutions, and $150 \mathrm{~min}$ of storage in cleansing solutions ${ }^{9}$.

The specimens were evaluated by using a $\mathrm{Re}$ flective spectrophotometer ${ }^{* * *}$ using the Commission Internationale de I'Eclairage (CIE). The aperture size was adjusted to $4 \mathrm{~mm}$ and the specimens were exactly aligned with the device. A white background was selected and measurements were made according to the CIE $\mathrm{L}^{*} \mathrm{a} * \mathrm{~b}^{*}$ color space relative to the CIE standard illuminant D65. Three readings were taken for each specimen, and mean values were calculated and recorded. The color changes $(\Delta \mathrm{E})$ of the specimens were calculated using the following formula:

$$
\Delta \mathrm{E}_{\mathrm{CIELAB}}=\left(\Delta \mathrm{L}^{* 2}+\Delta \mathrm{a}^{* 2}+\Delta \mathrm{b}^{* 2}\right) \frac{1 / 2}{2}
$$

Where: $L^{*}=$ lightness $(0-100), a^{*}=($ color change axis red/green) and $b^{*}=($ color change axis yellow/blue)

\footnotetext{
* Multisil-Epithetik, country (REF 540 01065) bredent GmbH \& Co.KG e-mail: R.D@ bredent.com.

* Nefertari Hair shampoo, Egypt,www.nefertaribodycare.com.

*** X-Rite, model RM200QC, Neu-Isenburg, Germany
} 


\section{RESULTS}

Data were presented as means and standard deviation (SD) values. Statistical analysis was performed with SPSS $^{*} 22^{\circledR}$ and Microsoft Excel ${ }^{* *}$ 2016. The results of this study were represented in tables (1), (2) and figure $(2,3)$. The significant level was set at $\mathrm{P} \leq 0.05$.

In this laboratory, study the comparison between the two different groups using Independent t-test while comparison between two successive follow up periods was performed by Paired t-test.

\section{Color change}

Paired t-test was performed to compare between color changes after $1 \& 2$ months. Regarding neutral

TABLE (1): Effect of time on color change for tested groups during follow up periods:

\begin{tabular}{|c|c|c|c|c|}
\hline & & $\begin{array}{c}\text { After one } \\
\text { month }\end{array}$ & $\begin{array}{l}\text { After } 2 \\
\text { months }\end{array}$ & $\mathbf{P}$ \\
\hline & & $\mathrm{M} \pm \mathrm{SD}$ & $\mathrm{M} \pm \mathrm{SD}$ & value \\
\hline \multirow{4}{*}{ 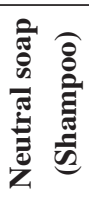 } & $\Delta \mathbf{L}$ & $0.98 \pm 0.23$ & $1.19 \pm 0.41$ & 0.06 \\
\hline & $\Delta \mathbf{a}$ & $0.58 \pm 0.17$ & $0.71 \pm 0.19$ & 0.08 \\
\hline & $\Delta \mathrm{b}$ & $0.28 \pm 0.08$ & $0.32 \pm 0.09$ & 0.14 \\
\hline & $\Delta \mathrm{E}$ & $1.81 \pm 0.21$ & $2.01 \pm 0.19$ & 0.07 \\
\hline \multirow{4}{*}{ 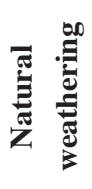 } & $\Delta \mathrm{L}$ & $1.52 \pm 0.29$ & $1.35 \pm 0.28$ & 0.09 \\
\hline & $\Delta \mathbf{a}$ & $0.73 \pm 0.25$ & $0.51 \pm 0.21$ & $0.005^{*}$ \\
\hline & $\Delta \mathrm{b}$ & $0.70 \pm 0.21$ & $0.58 \pm 0.19$ & 0.06 \\
\hline & $\Delta \mathrm{E}$ & $1.98 \pm 0.31$ & $2.21 \pm 0.48$ & $0.002 *$ \\
\hline
\end{tabular}

M; Mean, SD; Standard Deviation,

P; Probability level

*significant difference

$(\Delta E)=$ color changes of the specimens were evaluated using the following formula:

$\Delta E_{C I E L A B}=\left(\Delta L^{2}+\Delta a^{2}+\Delta b^{2}\right)^{1 / 2}$

$\Delta L=$ lightness (0-100),

$\Delta a=$ (change the color of the axis red/green)

$\Delta b=($ color variation axis yellow/blue $)$ soap (shampoo) it was noted that, $\Delta \mathrm{L}, \Delta \mathrm{a}, \Delta \mathrm{b}$ and $\Delta \mathrm{E}$ values after 2 months was insignificantly higher than after 1 months $(\mathrm{P}>0.05)$. While in natural weathering it was noted that $\Delta \mathrm{E}$ values after 2 months was significantly higher than after 1 months $(\mathrm{P}<0.05)$. However, $\Delta \mathrm{L}$ and $\Delta \mathrm{b}$ values after 2 months were insignificantly lower than after 1 month $(\mathrm{P}>0.05)$ and $\Delta \mathrm{a}$ was significantly lower after 2 months as presented in table (1) and Fig. (2).

Also Independent T-test analysis was used to compare between shampoo and natural weathering which reveals significant difference regarding $\Delta \mathrm{L}$, $\Delta \mathrm{a}, \Delta \mathrm{b}$ and $\Delta \mathrm{E}$ after 1 months ( $\mathrm{P}$ value $<0.05$ ), and revealed significant difference regarding $\Delta \mathrm{a}, \Delta \mathrm{b}$ and $\Delta \mathrm{E}$ after 2 months $(\mathrm{P}$ value $<0.05)$ as presented in table (2) and Fig. (3).

TABLE (2): Comparison of color change between the tested groups:

\begin{tabular}{|c|c|c|c|c|}
\hline & & $\begin{array}{c}\text { Neutral soap } \\
\text { (Shampoo) }\end{array}$ & $\begin{array}{c}\text { Natural } \\
\text { weathering }\end{array}$ & D volu \\
\hline & & $\mathrm{M} \pm \mathrm{SD}$ & $\mathrm{M} \pm \mathrm{SD}$ & P value \\
\hline & $\Delta \mathrm{L}$ & $0.98 \pm 0.23$ & $1.52 \pm 0.29$ & $0.001 *$ \\
\hline $\bar{E}$ & $\Delta \mathbf{a}$ & $0.58 \pm 0.17$ & $0.73 \pm 0.25$ & $0.001 *$ \\
\hline 远 & $\Delta \mathrm{b}$ & $0.28 \pm 0.08$ & $0.70 \pm 0.21$ & $0.001^{*}$ \\
\hline & $\Delta \mathbf{E}$ & $1.81 \pm 0.21$ & $1.98 \pm 0.31$ & $0.04 *$ \\
\hline & $\Delta L$ & $1.19 \pm 0.41$ & $1.35 \pm 0.28$ & 0.15 \\
\hline$\stackrel{n}{\tilde{c}}$ & $\Delta \mathbf{a}$ & $0.71 \pm 0.19$ & $0.51 \pm 0.21$ & $0.003^{*}$ \\
\hline$\overline{\mathrm{o}}$ & $\Delta \mathrm{b}$ & $0.32 \pm 0.09$ & $0.58 \pm 0.19$ & $0.03 *$ \\
\hline & $\Delta E$ & $2.01 \pm 0.19$ & $2.21 \pm 0.48$ & $0.03^{*}$ \\
\hline
\end{tabular}

M; Mean, SD; Standard Deviation, P; Probability level *significant difference $(\Delta E)=$ color changes of the specimens were evaluated using the following formula:

$\Delta E_{C I E L A B}=\left(\Delta L^{2}+\Delta a^{2}+\Delta b^{2}\right)^{1 / 2}$

$\triangle L=$ lightness (0-100), $\Delta a=$ (change the color of the axis red/green) $\Delta b=($ color variation axis yellow/blue $)$

\footnotetext{
* Statistical Package for Social Science, IBM, USA

** Microsoft Co-operation, USA.
} 


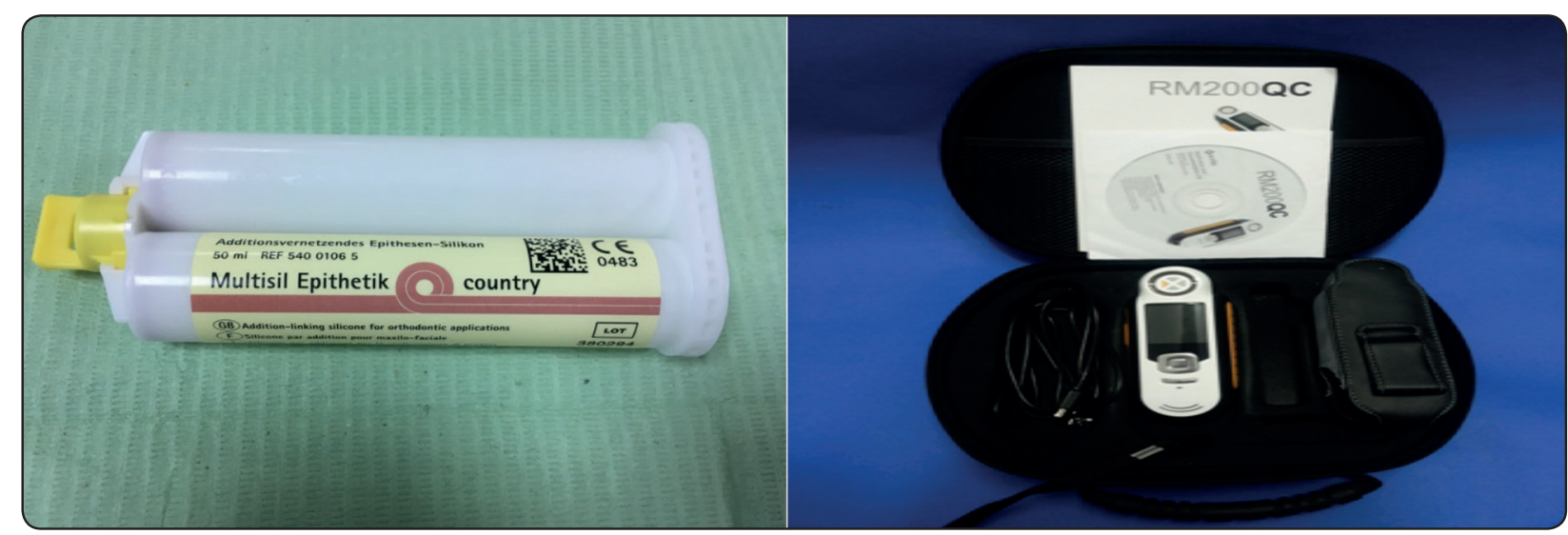

Fig. (1): Maxillofacial silicone and the spectrophotometer.

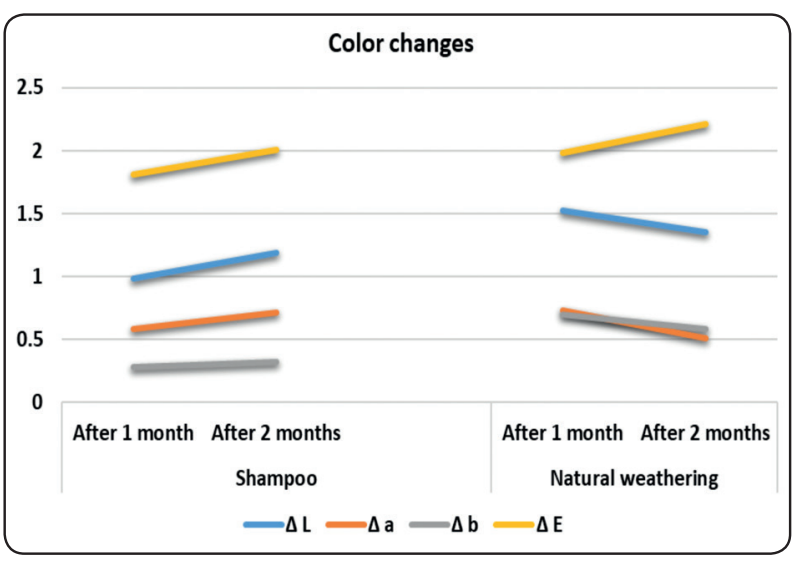

Fig. (2): Line chart represents effect of time on color change for tested groups during follow- up

\section{DISCUSSION}

The most significant parameter in evaluating the performance of facial prostheses is color. Color is the major concern for the patient whether using the prosthesis or dislike and switch to a new prosthesis. Alteration of the physical properties is the main reason for color fading and disliking their prostheses. The major cause for the limited service of maxilofacial prosthesis is color change and the quick deterioration of the silicon elastomer. ${ }^{1}$

Chromatic alteration is usually caused by intrinsic and extrinsic factors. The intrinsic factors include discoloration of the material itself with matrix alterations. This commonly occurs after aging

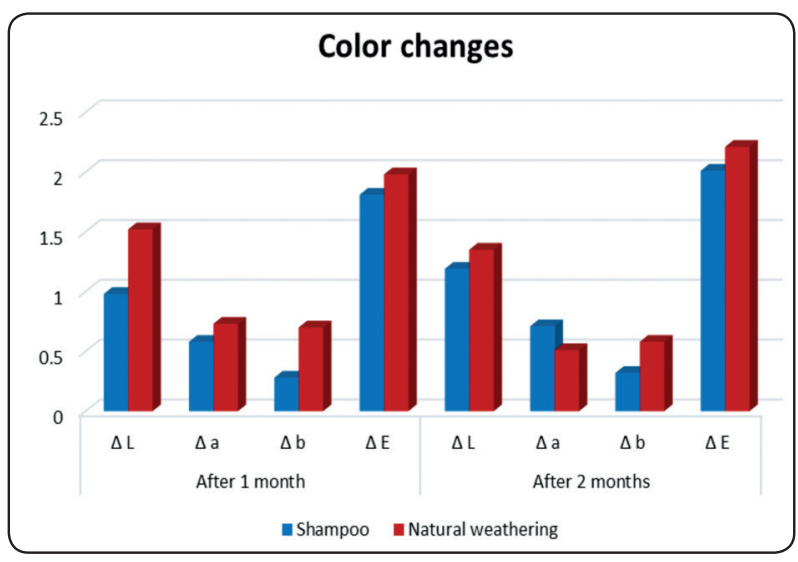

Fig (3): Bar chart represents color change of two groups during follow up

because of some physical-chemical conditions such as variation in humidity and thermal conditions. Extrinsic factors, such as the absorption and adsorption of materials may generate discoloration. Other factors as accumulation of stains, water absorption, dehydration, infiltration, chemical degradation, degradation from use, surface roughness, oxidation during double carbon reactions to produce peroxide compounds, and continuous formation of pigments due to degradation of products contribute to the alterations that occur. ${ }^{13}$

The success of any maxillofacial prosthesis depends on the physical and mechanical properties of the material used in its construction, consequently 
conducting many tests to evaluate the properties of the facial prosthesis including color stability, surface roughness tests, and scanning microscope analysis. The spectrophotometer was well suitable for determining any minor variations in the color of the elastomers, it has numerous benefits such as it being adaptable and versatile: used for lab, or field operation; easy to read; quick color compare and certificates quick measurement. ${ }^{14-16}$

Commission Internationale de l'Eclairge, L*, $a^{*}, b^{*}$ (CIELAB) perceptibility and acceptability thresholds for light skin-colored maxillofacial silicone specimens are 1.1 and 3.0, respectively. Color changes greater than 3 are considered visual by the naked eye. $\Delta \mathrm{E}$ of 1.1 and 3.0 were selected as estimated for the incidence of minimum satisfactory color changes. In our study $\Delta \mathrm{E}$ for neutral soap and natural weathering after 2 months was 2.01 and 2.21 respectively, which is acceptable color change. Only $\Delta \mathrm{E}$ values higher than 3 can be detected by the human eye. This indicates that the material used in this study can withstand color change against natural weathering conditions and we also recommend using the neutral soap type that was used in this study. ${ }^{8,10,12}$

Several authors suggested that color change may undergo with time without any external factor, which may be attributed to the additional cross-linking caused by continued chemical polymerization of the elastomer or by side reactions among impurities present within the silicone. Therefore it is worthy that the clinician must know the pigments, the coloration methods, and polymerization procedures. Also the nonstop release of sub-products throughout this continuous polymerization process not only causes dimensional alterations of the silicon material but also chromatic pattern variations. ${ }^{10,16}$

Color deterioration of the maxillofacial silicone prosthesis is attributed to the collective effect of numerous aspects such as environmental exposure, UV radiation, humidity, air pollutants, the method of disinfection and exposure to facial secretions as perspiration. On the other side internal factors include silicone composition, mode of curing, degree of cross-linking, extrinsic and intrinsic stains used; all these factors have a significant role in preserving or deteriorating the color of the silicone prosthesis. ${ }^{9}$

Neutral soap removes nanoparticles (pigments) from superficial layer of silicone since this method depends on mechanical technique such as digital friction, scrubbing and brushing. This fact can justify color alteration presented in specimens of group disinfected with neutral soap which is insignificant. ${ }^{1,4}$

Although the neutral soap hygiene procedure necessities friction of the samples, it was less destructive than the immersion technique used for $1 \%$ hypochlorite. The neutral soap is considered chemically inert. Surveys of previous studies have reported that, the hygiene procedure with neutral soap is less destructive to maxilofacial silicone when compared to $1 \%$ hypochlorite even when using extrinsic pigmentation..$^{8,17,18}$

An in-vitro study emphasizes that immersion of the samples in neutral soap solution produced the least color change regardless of the material used other studies recommended using mild detergents for cleaning facial prosthesis. The neutral soap used in this study was commercially available with ph 7.6.9 Specimens treated with neutral soap induced clinically acceptable color change.

Exposure to UV light alters the color of the elastomers due to intrinsic chemical modifications in silicon or discoloration of some pigments that are not UV resistant. Photo-oxidation and hydrolysis of the silicone elastomer are the major deterioration reactions that happen following exposure to sunlight, temperature, humidity, and air pollutants existing in the atmosphere surrounding the specimens. These reactions produce changes in physical and chemical properties resulting in deterioration of the silicone. ${ }^{1,4}$ 
In addition, silicone facial prosthetics are often associated with color changes owing to their porous and irregular surface. Surface roughness is frequently a good indicator of the performance of the silicon material. Many studies emphasize that surface roughness was significantly increased after natural weathering which may be due to small cracks and some irregularities on the surface with reduction in the hydrophobicity by the effect of the weather conditions or may be due to high filler concentration in the material composition, which promoted higher roughness. The irregularities in the surface allow collection of microscopic debris in the pores that causes chromatic alterations., ${ }^{1,19}$

This significant color change at $\Delta \mathrm{a}$, and $\Delta \mathrm{E}$ at natural weathering group can be attributed to the existence of UV light irradiation present in the solar radiation which may have enhanced cross-linking, along with accelerated interaction of the fatty acids with silicone, destroying the chain bonds, and decomposing the elastomer. The results obtained in our study showed that the magnitude of color changes $(\Delta E=2.21)$ in the tested natural weathering groups which is within the estimated range chosen for the occurrence of minimum acceptable color changes. This might be due to the existence of surface treated hydrophobic silica fillers present in the polymer matrix repelling water molecules and hence prevent solution absorption into the material. Furthermore, the hydrophobic character of silicone matrix is enhanced, with the addition of pigments because of the vinyl functional silanes groups present in their chemical structure..$^{9,14}$

The ideal elastomer colorant combination should not only allow satisfactory esthetics to be achieved clinically, but should also maintain the esthetics and physical properties indefinitely, or at least until the patient's tissues have changed to the point that fit the prosthesis. ${ }^{20}$

Addition type of polymer has less color change when compared to condensation type, it could be attributed to high filler content, lower opacity, ${ }^{1,9}$ which can explain the acceptable color change range of the material when exposed to natural weathering and neutral soap (shampoo).

\section{CONCLUSIONS}

The room temperature silicone based on addition linking vinyl polydimethylsiloxanes, fillers, pigments and platinum complexes, final hardness 35 shore $\mathrm{A}$ has acceptable cross-linked formulation that withstands natural weathering conditions and neutral soap disinfection. Within the limitations of this study, the results showed that exposure of silicon to natural weathering had greater effect on color change than neutral soap (shampoo) group. But the magnitude of color change in both groups is within the estimated range chosen for the occurrence of minimum acceptable color changes according to Commission Internationale de l'Eclairge, $\mathrm{L}^{*}$, $a^{*}, b^{*}($ CIELAB) perceptibility and acceptability thresholds for light skin-colored maxillofacial silicone specimens.

\section{REFERENCES:}

1. Babu, A. S., Manju, V. \& Gopal, V. K. Effect of chemical disinfectants and accelerated aging on maxillofacial silicone elastomers: An In vitro Study. Indian J. Dent. Res. 29, 67-73 (2018).

2. Cifter, E. D. et al. Effect of vulcanization temperature and dental stone colour on colour degradation of maxillofacial silicone elastomers. BMC Oral Health 17, (2017).

3. Cifter, E. D. et al. In vitro study of effects of aging and processing conditions on colour change in maxillofacial silicone elastomers. BMC Oral Health 19, (2019).

4. Manjula, N. Effect of denture cleansers and accelerated aging on the color stability of maxillofacial silicone: An in vitro study. Indian J. Multidiscip. Dent. 7, 110 (2017).

5. Reddy, Jr., Kumar, Bm., Ahila, S. \& Rajendiran, S. Materials in maxillo-facial prosthesis. J. Indian Acad. Dent. Spec. Res. 2,1 (2015).

6. Chamaria, A., Aras, M. A., Chitre, V. \& Rajagopal, P. Effect of Chemical Disinfectants on the Color Stability of Maxillofacial Silicones: An In Vitro Study. J. Prosthodont. 28, e869-e872 (2019). 
7. Montgomery, P. C. \& Kiat-Amnuay, S. Survey of currently used materials for fabrication of extraoral maxillofacial prostheses in North America, Europe, Asia, and Australia. J. Prosthodont. 19, 482-490 (2010).

8. Pesqueira, A. A. et al. Effect of Disinfection and Accelerated Aging on Color Stability of Colorless and Pigmented Facial Silicone. J. Prosthodont. 20, 305-309 (2011).

9. Mehta S, N. D. A. A spectrophotometric analysis of extraoral aging conditions on the color stability of maxillofacial silicone. J Indian Prosthodont Soc. (4), 355-360 (2017).

10. Bankoğlu, M., Oral, I., Gül, E. B. \& Yilmaz, H. Influence of pigments and pigmenting methods on color stability of different silicone maxillofacial elastomers after 1-year dark storage. J. Craniofac. Surg. 24, 720-724 (2013).

11. Johnston, W. M. Color measurement in dentistry. Journal of Dentistry https://dx.doi.org/10.1016/j.jdent.2009.03.011 (2009) doi:10.1016/j.jdent.2009.03.011.

12. CIE, I. C. on I. C. Official Recommendations of the International Commission on Illumination. Publication CIE No. 15 (E-1.3.1). 210-215 (1976) doi:10.1364/ JOSA.64.000210.

13. Mancuso, D. N., Goiato, M. C., De Carvalho Dekon, S. F. \& Gennari-Filho, H. Visual evaluation of color stability after accelerated aging of pigmented and nonpigmented silicones to be used in facial prostheses. Indian J. Dent. Res. 20, 77-80 (2009).
14. Al-Dharrab, A. A., Tayel, S. B. \& Abodaya, M. H. The Effect of Different Storage Conditions on the Physical Properties of Pigmented Medical Grade I Silicone Maxillofacial Material. ISRN Dent. 2013, 1-9 (2013).

15. Douglas, R. D., Steinhauer, T. J. \& Wee, A. G. Intraoral determination of the tolerance of dentists for perceptibility and cceptability of shade mismatch. J. Esthet. Restor. Dent. 21, 133-134 (2009).

16. Hatamleh, M. M., Polyzois, G. L., Silikas, N. \& Watts, D. C. Effect of extraoral aging conditions on mechanical properties of maxillofacial silicone elastomer. J. Prosthodont. 20, 439-446 (2011).

17. Cabral, Y. M., Pesqueira, A. A., Moreno, A., Goiato, M. C. \& Haddad, M. F. Effect of different methods of hygiene on the color stability of extrinsically pigmented facial silicone. Brazilian J. Oral Sci. 17, 17 (2018).

18. Goiato, M. C. et al. Effect of chemical disinfection and accelerated aging on color stability of maxillofacial silicone with opacifiers. J. Prosthodont. 20, 566-569 (2011).

19. El Afandy, H., Fawzy, A. Evaluation of mechanical properties of maxillofacial silicone after long term exposure to different conditions. Egypt. Dent. J. 65, 2681-2689 (2019).

20. Cevik, P. \& Yildirim-Bicer, A. Z. Effect of different types of disinfection solution and aging on the hardness and Colour stability of maxillofacial silicone elastomers. Int. J. Artif. Organs 41, 108-114 (2018). 